\title{
Möglicherweise Zeichen eines Malignoms
}

\section{Die Dermatomyositis ist primär eine dermatologische Erkrankung. Sie manifestiert sich mit charakteristischen Hauterscheinungen. Etwa 30\% der Patienten haben ein Malignom (paraneoplastische Dermatomyositis).}

$D$ ie Dermatomyositis ist eine multifaktoriell bedingte systemische Autoimmunerkrankung. Ätiologisch werden virale Infekte oder auch Medikamente beschrieben. Frauen sind etwa doppelt so häufig betroffen wie Männer. Ein zweiter Manifestationsgipfel liegt zwischen 55 und 60 Jahren. Dieser ist häufig paraneoplastisch induziert. In vielen Fällen zeigt sich eine allgemeine Abgeschlagenheit und geringere Belastbarkeit. Die Patienten berichten, alltägliche Arbeiten wie Fensterputzen, Treppensteigen o. ä. aufgrund von muskulären Beschwerden nicht mehr durchführen zu können.

Klinisch imponieren einige spezifische Zeichen, die in einem Drittel der Fälle auch den muskulären Beschwerden zuvorkommen. Diagnostisch wegweisend sind die sogenannten Gottron-Papeln (Abbildung 1). Sie zeigen sich auf lividroten Erythemen und tastbaren Papeln über den Streckseiten der Finger. Auch Nagelfalzveränderungen können auftreten; ein Zurückziehen des Nagelfalzes ist häufig schmerzhaft und wird als KeinigZeichen beschrieben. Faszial findet sich bei diesen Patienten besonders initial eine Gesichtsschwellung mit Lidödem, des Weiteren werden auch teils schmerzhafte Erytheme schmetterlingsartig an den Wangen unter Aussparung der perioralen Bereiche bis hin zur Halsregion beobachtet (Shawl-Zeichen, Abbildung 2). Im weiteren Verlauf konfluiert das Gesamtbild zu einem poikilodermatischem Befund mit Atrophien und auch sklerotischen Arealen. Im Rahmen des systemischen Krankheitsbildes zeigen sich bei der Dermatomyositis progrediente Muskelschwächen speziell der proximalen Muskulatur mit der Gefahr der Kalzifikation. Bei einem Viertel der Patienten kommt es zu einer Gelenkbeteiligung. Pneumonien zeigen sich in bis $\mathrm{zu} 20 \%$ aller Patienten. Serologisch zeigt sich eine Erhöhung der Kreatininphosphokinasase, in einem Drittel der Fälle finden sich positive antinukleäre Antikörper. Die Diagnosesicherung erfolgt in der Zusammenschau der Klinik, Histologie und des Elektromyogramms.

Differentialdiagnostisch müssen ein Lupus erythematodes und andere Bindegewebserkrankungen abgegrenzt werden. Im Rahmen des Stagings ist speziell bei älteren Patienten ein Tumorausschluss obligat. Speziell Tumoren der Ovarien, der Lunge, des Pankreas und des Gastrointestinaltrakts werden beschrieben. Auch ein Non-Hodgkin-Lymphom muss bedacht werden. Schwierig bleibt die sichere Einordnung der Dermatomyositis als paraneoplastisch induziert, da sie auch Wochen bis Monate vor der Diagnosestellung der zugrunde liegenden Neoplasie klinisch manifest werden kann. Deshalb sollten alle älteren Patienten mit Dermatomyositis weiterbeobachtet werden, um potenzielle Neoplasien so früh wie möglich zu diagnostizieren.

Im Mittelpunkt der Behandlung steht neben den zugrundeliegenden Triggerfaktoren eine systemische immunsuppressive Therapie, initial mit systemischen Steroiden und im weiteren Verlauf mit MTX, Ciclosporin oder auch Cyclophosphamid, um Steroide einzusparen. Rituximab zeigte in Studien ebenfalls ein gutes Ansprechen. Die Prognose der Dermatomyositis ist abhängig von der zugrunde liegenden Ätiologie, als paraneoplastisches Geschehen ist sie schlecht.

Dr. med. Andreas Körber Klinik und Poliklinik für Dermatologie, Venerologie und Allergologie der Universität Essen

Hufelandstraße 55, 45122 Essen

E-Mail: Andreas.Koerber@uk-essen.de

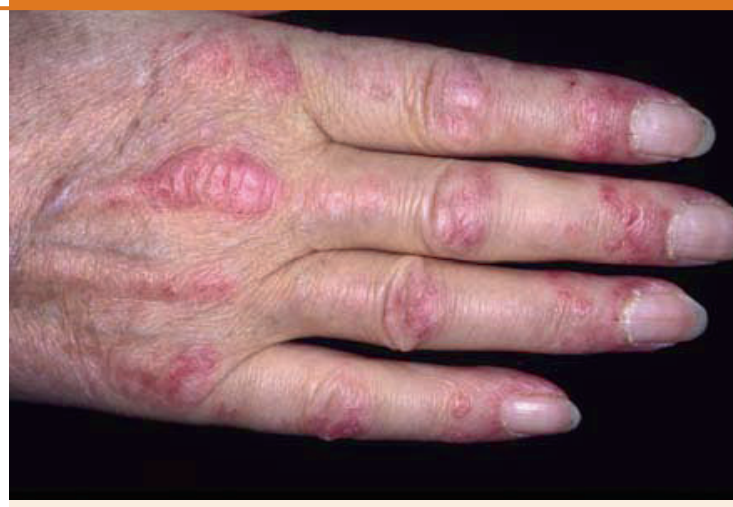

Abbildung 1. Gottron-Papeln

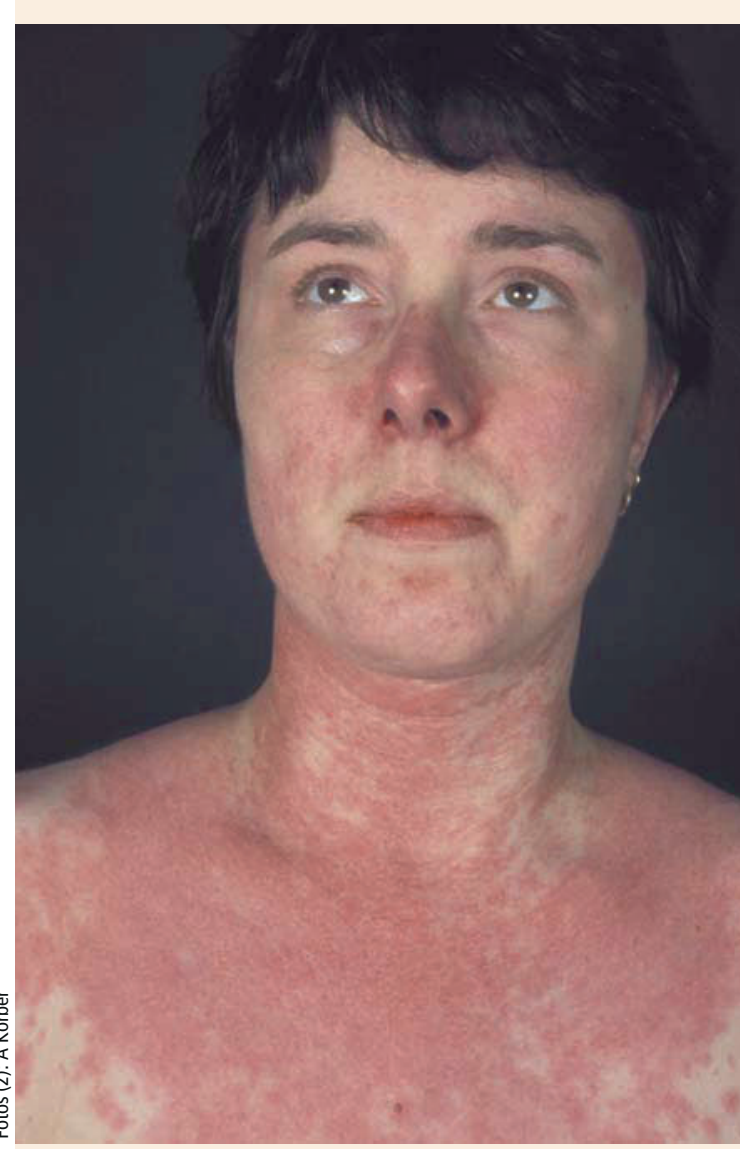

Abbildung 2. Shawl-Zeichen

\section{Ihr besonderer Fall?}

Sicher sehen auch Sie ab und an einen eindrucksvollen Befund in Ihrer Praxis oder Klinik. Fotografieren Sie ihn, schreiben Sie uns unter dem Stichwort „Onkologie im Bild“, bei Veröffentlichung erhalten Sie 100 Euro. Redaktion InFo Onkologie

E-Mail: doris.berger@springer.com Fax: 089/203043-1400 